\title{
"Double-trace" Deformations, Boundary Conditions and Spacetime Singularities
}

\author{
Micha Berkooz $a$, 1, Amit Severb, 目 and Assaf Shomerb, 3 \\ ${ }^{a}$ Department of Particle Physics, The Weizmann Institute of Science, Rehovot 76100, Israel \\ ${ }^{b}$ Racah Institute of Physics, The Hebrew University, Jerusalem 91904, Israel \\ Double-trace deformations of the AdS/CFT duality result in a new perturbation ex- \\ pansion for string theory, based on a non-local worldsheet. We discuss some aspects of \\ the deformation in the low energy gravity approximation, where it appears as a change in \\ the boundary condition of fields. We relate unique features of the boundary of $A d S$ to the \\ worldsheet becoming non-local, and conjecture that non-local worldsheet actions may be \\ generic in other classes of backgrounds.
}

January 2002

1 E-mail : Micha.Berkooz@weizmann.ac.il. Incumbent of the Recanati career development chair of energy research.

2 E-mail : asever@cc.huji.ac.il .

3 E-mail: shomer@cc.huji.ac.il. 


\section{Introduction}

Non-local string theories [1.2] (NLST's) are new string theories with the following properties

1. They are described by a non-local worldsheet action.

2. The familiar string perturbation theory is modified. In addition to the usual topologies there are now singular topologies, weighted with a new expansion parameter. This new parameter is not a modulus.

3. The theories are maximally non-local on parts of the space. These theories appear as deformations of the familiar $A d S \times \mathcal{M}$ backgrounds, and the theory is generically maximally non-local on $\mathcal{M}$ in the sense that it singles out a unique low-lying eigenvalue of the Laplacian on $\mathcal{M}$ and changes only the interactions of the corresponding mode.

These theories come about as deformations of ordinary string theories on $A d S$ spaces, via the AdS/CFT duality [3, 国, (for a review see [6]). The spectrum of fields on the gravitational side of the duality corresponds to a subset of the operators in the dual CFT these are usually referred to as "single trace operators". Other operators that appear in the OPE of the single trace operators are described by multi-particle states in the gravitational dual. These operators are referred to as "multi-trace operators". NLST's come about when there are marginal and relevant operators that are multi-trace operators 1 . Deforming the boundary theory by such operators does not correspond to any known stringy small shift of the background (since these are all associated with single particle states). Hence the theory is deformed in a new way. In [1] it was shown that the familiar notions of string perturbation theory themselves need to be modified, leading to properties 1-3 above.

In this paper we will elaborate on how this deformation effects the low energy effective action. We will show, starting from the formulation in [1] of the deformation using an auxiliary variable, that the boundary conditions on some fields change. This is a significant change in gravity, where determining the boundary conditions is at times a subtle task, which influences physical observables. Whereas this issue is usually neglected in smooth, asymptotically flat spaces (where they play a small role), they are very important in other cases such as $A d S$ and spaces with singularities - particularly cosmological singularities.

Motivated by this we are led to speculate what might be the more general relation

4 The truly marginal deformation on $A d S$ is the main case studied so far. NLST's and Multitrace deformations are, however, expected to play an important role in the relevant deformation cases as well [7]. In this paper we will discuss a marginal but not truly marginal deformation 
between NLST's and qualitative features of spacetimes. We suggest that NLST may be relevant in cases where there are severe enough singularities, defects or perhaps horizons, at finite distance or time (or finite affine parameter) in spacetime. If this conjecture turns out to be true, one might need to deal more extensively with NLST's to understand such backgrounds.

In section 2 we discuss the gravity limit of the usual AdS/CFT correspondence. Our presentation will be slightly different from the usual presentation. This treatment is chosen because it is better adapted to the subsequent discussion of double trace deformations 5 . In section 3 we discuss the change in the boundary conditions which occurs when deforming the theory by a double trace deformation. The double trace deformation which we have chosen is the simplest one, a deformation by : $O^{2}$ : where $O$ is an operator of dimension 6 $d / 2$. In section 4 , which is more speculative, we try to relate the fact that the double trace deformation changes the boundary conditions to the non-locality of the worldsheet, and we argue that generally when there are singularities at a finite distance or time in spacetime, such as the boundary of AdS, the theory will be non-local on the worldsheet. Boundary conditions in $A d S / C F T$ correspondence where recently discussed in [8]. In [9] Witten reaches a similar conclusion regarding the change of boundary conditions in the presence of a double-trace deformation.

\section{AdS/CFT correspondence, the usual case.}

In this section we review the familiar way in which one computes correlators using the AdS/CFT correspondence. We will set-up the procedure in a slightly different form than usual, that will be more convenient when we discuss the double trace deformation. The differences go away when we remove the IR regulators in $A d S$.

Subsection 2.1 includes the basic set-up from the literature, 2.2 discusses the modification that we need and set up notations for the rest of the paper, and section 2.3 discusses our formulation in the presence of sources on the boundary.

\subsection{Basic setup}

According to the AdS/CFT correspondence [3,4,5] (for a review see [6]), the generating functional of correlation functions for some operator $\mathcal{O}$ in the CFT dual to an AdS

5 The differences are expected to disappear as the IR cut-off in $A d S$ is removed.

6 : $O^{2}$ : will turn out to be marginal but not truly marginal. 
geometry, is equal to the partition function of string theory in that background, with specific behavior of the fields near the boundary. We will primarily work in the Kaluza-Klein reduction down to $A d S_{d+1}$ and suppress the compact internal manifold and the 10 or 11 dimensional picture. We will also focus on the case of a single real scalar field $\phi$ on $A d S_{d+1}$.

Imposing boundary conditions $\rho$ for $\phi$ (this will be made more precise below, adapted for our purposes), the relation is written as

$$
Z[\rho]_{C F T} \equiv<e^{\int \rho A}>_{C F T}=Z[\rho]_{\text {string theory }}
$$

The quantity on the RHS is the full stringy partition function. Since we are interested only in the dynamics of one scalar field we will slightly abuse notation and write it as if it is a field theory path integral over this scalar field. This is justified at low enough energies before quantum gravity and stringy effects set in, which is the regime we are interested in. Hence we will write the correspondence as

$$
Z[\rho]_{C F T} \equiv<e^{\int \rho A}>_{C F T}=\int D[\phi ; \rho] e^{-\mathcal{S}_{g r}[\phi]},
$$

where $D[\phi ; \rho]$ stands for a path integral over the field $\phi$ with boundary conditions set by $\rho$, and $\mathcal{S}_{g r}$ is the gravity action.

We work in Euclidean $A d S$ in the Poincaré patch with coordinates:

$$
d s^{2}=\frac{d z^{2}+d x_{i} d x_{j} \delta^{i j}}{z^{2}}, \quad i, j=1 \ldots d
$$

and we have set the length scale of $A d S$ to 1 . The action of a scalar field with mass $m$ (where the Breitenlohner-Freedman bound restricts $m^{2} \geq-\frac{d^{2}}{4}$ ) in these coordinates is:

$$
\mathcal{S}_{0}=-\frac{1}{2} \int d^{d} x d z \phi\left[\partial_{z} z^{-d+1} \partial_{z}+z^{-d+1} \partial_{i} \partial^{i}-z^{-d-1} m^{2}\right] \phi
$$

The general solution to the equations of motion derived from (2.4) for a scalar field with mass $m^{2}>-\frac{d^{2}}{4}$ is 10,4 :

$$
\phi(\vec{x}, z)=\int_{\partial A d S} d^{d} x^{\prime} \frac{\Sigma^{-}\left(\vec{x}^{\prime}\right) z^{\Delta_{+}}}{\left(z^{2}+\left|\vec{x}-\vec{x}^{\prime}\right|^{2}\right)^{\Delta_{+}}}+\int_{\partial A d S} d^{d} x^{\prime} \frac{\Sigma^{+}\left(\vec{x}^{\prime}\right) z^{\Delta_{-}}}{\left(z^{2}+\left|\vec{x}-\vec{x}^{\prime}\right|^{2}\right)^{\Delta_{-}}},
$$

where $\Delta_{ \pm}=\frac{d}{2} \pm \sqrt{\left(\frac{d}{2}\right)^{2}+m^{2}}$, and $\Sigma^{ \pm}$are arbitrary functions of $x$. Using the fact that

$$
\lim _{z \rightarrow 0} \frac{z^{2 \Delta_{+}-d}}{\left(z^{2}+\left|\vec{x}-\vec{x}^{\prime}\right|^{2}\right)^{\Delta_{+}}}=\pi^{\frac{d}{2}} \frac{\Gamma\left(\Delta_{+}-\frac{d}{2}\right)}{\Gamma\left(\Delta_{+}\right)} \delta^{d}\left(\vec{x}-\vec{x}^{\prime}\right),
$$


the asymptotic behavior near the boundary is

$$
\phi(z, \vec{x}) \sim \phi_{0}(\vec{x}) z^{\Delta_{-}}\left(1+O\left(z^{2}\right)\right)+A(\vec{x}) z^{\Delta_{+}}\left(1+O\left(z^{2}\right)\right)
$$

where $\phi_{0}$ and $A$ are known linear functionals of $\Sigma^{-}$and $\Sigma^{+}$.

In the degenerate case $\Delta_{+}=\Delta_{-}=\frac{d}{2}$ (i.e. $m^{2}=-\frac{d^{2}}{4}$ ) additional regularization is required and the second independent solution of (2.5) has a logarithmic term.

\subsection{Classical solutions and boundary conditions}

In order to allow for classical solutions to (2.4), such as (2.5), one must fix appropriate boundary conditions. The linear variation of (2.4) is:

$$
\delta \mathcal{S}^{(1)}=\int_{A d S} d^{d+1} x \sqrt{g} \delta \phi\left[-\nabla^{2}+m^{2}\right] \phi+\frac{1}{2} \int_{\partial(A d S)} d^{d} x z^{-d+1}\left(\phi \partial_{z} \delta \phi-\left(\partial_{z} \phi\right) \delta \phi\right) .
$$

The first term vanishes if $\phi(\vec{x}, z)$ obeys the equations of motion in the bulk. The second term vanishes if one fixes the following boundary condition 7 :

$$
\left.z \partial_{z} \phi\right|_{\partial}=\left.\omega \phi\right|_{\partial}
$$

where $\omega$ is an arbitrary function, naturally taken to be a constant, and $\partial$ stands for $\partial(A d S)$ - the boundary of $A d S$. This restricts the variation on the boundary to obey:

$$
\left.z \partial_{z} \delta \phi\right|_{\partial}=\left.\omega \delta \phi\right|_{\partial}
$$

Plugging the general solution (2.5) into (2.9) where we set the boundary at some finite cutoff $8=\epsilon$ gives a complicated non-local integral relation between $\Sigma^{+}$and $\Sigma^{-}$In the limit $\epsilon \rightarrow 0$ we can use (2.7) instead of (2.5) and this relation simplifies to the following local expression:

$$
\left[\left(\Delta_{-}-\omega\right) \phi_{0}(\vec{x}) \epsilon^{\Delta_{-}}+\left(\Delta_{+}-\omega\right) A(\vec{x}) \epsilon^{\Delta_{+}}\right]\left(1+\mathcal{O}\left(\epsilon^{2}\right)\right)=0
$$

7 Note that this is not the most general boundary condition possible. We will shortly present a more general boundary condition.

8 We are imposing a cut off near $z=0$ in the Euclidean Poincaré patch. One needs also to regulate $z \rightarrow \infty$ since it is also a boundary point of the geometry. This can be taken care of by requiring that all sources on the boundary decay fast enough at $|\vec{x}| \rightarrow \infty$. 
There are two special choices that simplify this relation furtherl. If we take $\omega=\Delta_{ \pm}$ we can write (2.9) as:

$$
\left[z^{-\Delta_{\mp}}\left(z \partial_{z}-\Delta_{ \pm}\right) \phi(\vec{x}, z)\right]_{\partial}=0
$$

where we multiplied (2.11) by $z^{-\Delta_{\mp}}$ to obtain a finite relation near $z \sim 0$. In the limit $\epsilon \rightarrow 0$ this fixes the value of $\phi_{0}=0(A=0)$ but puts no restriction on $A\left(\phi_{0}\right)$, thus recovering the usual interpretation of $A\left(\phi_{0}\right)$ as fluctuating (in the Euclidean path integral [10]) and $\phi_{0}(A)$ as a constant background. This was the case where the classical source on the boundary is set to zero. We will now modify the boundary condition so as to include a non-zero source.

\subsection{Adding a classical source - finite cutoff treatment.}

The boundary condition (2.9) makes sure that the integrand $\left[\phi \partial_{z} \delta \phi-\left(\partial_{z} \phi\right) \delta \phi\right]$ vanishes on the boundary. However, we can relax this condition and demand only that the whole integral $\int_{\partial} d^{d} x z^{-d+1}\left(\phi \partial_{z} \delta \phi-\left(\partial_{z} \phi\right) \delta \phi\right)$ will vanish on the boundary. The general boundary condition assuring this is:

$$
\left.z \partial_{z} \phi(\vec{x}, z)\right|_{\partial}=\int_{\partial} d^{d} x^{\prime} \Omega\left(\vec{x}, \vec{x}^{\prime}\right) \phi\left(\vec{x}^{\prime}, z\right),
$$

where $\Omega\left(\vec{x}, \vec{x}^{\prime}\right)$ is symmetric in $x$ and $x^{\prime}$. The generalization to $\Omega$ will be important below when we discuss the double trace deformation. Usually one takes $\Omega=0$ for $\vec{x} \neq \vec{x}^{\prime}$. We will relax this here. This is reasonable for the following two reasons:

1. We will require that the support of $\Omega$ shrinks to $\vec{x}=\vec{x}^{\prime}$ when we remove the cutoff from $A d S$. Hence it is again, from the dual field theory point of view, merely a different regularization scheme.

2. Reinstating the compact manifold that multiplies $A d S$, our deformation will be highly non-local in it, so we have already given up locality to some extent anyhow.

For later convenience we will use infinite matrix notations, where integrals are written as summation of indices,

$$
\int_{z=\epsilon} d^{d} x d^{d} x^{\prime} \chi(\vec{x}, z) F\left(\vec{x}, \vec{x}^{\prime}\right) \psi\left(\vec{x}^{\prime}, z\right) \equiv \chi_{\vec{x}} \mathbf{F}_{\vec{x}^{\prime}}^{\vec{x}^{\prime}} \psi^{\vec{x}^{\prime}} \equiv \chi^{T} \mathbf{F} \psi
$$

9 This is different then the more familiar formulation where one imposes Dirichlet boundary conditions on the scalar field. The two are just different regularization schemes. The conventions we adopt here will be more useful below. 
Note that the evaluation of the integral at the boundary $z=\epsilon$ is implicit in our notation. The boundary condition (2.13) will now be written as $\left.z \partial_{z} \phi\right|_{z=\epsilon}=\boldsymbol{\Omega} \phi$.

We can now write down the boundary condition with an external source as:

$$
\left.z^{-\Delta}\left(z \partial_{z}-\mathbf{\Omega}\right) \phi\right|_{z=\epsilon}=\mathbf{A} \rho
$$

where $\boldsymbol{\Omega}$ is as above and $\mathbf{A}$ is an arbitrary linear functional, which encodes the renormalization of the source with the cut-off $\epsilon$. We will make a convenient choice for $\mathbf{A}$ below, but different choices of $\mathbf{A}$ amount again to different regularization schemes. (2.15) restricts the fluctuations to obey $\left.z^{-\Delta}\left(z \partial_{z}-\boldsymbol{\Omega}\right) \delta \phi\right|_{z=\epsilon}=0$ so that now the linear variation (2.8) is:

$$
\delta \mathcal{S}^{(1)}=-\frac{1}{2} \int_{z=\epsilon} d^{d} x d^{d} x^{\prime} z^{\Delta-d} \delta \phi(\vec{x}, z) A\left(\vec{x}, \vec{x}^{\prime}\right) \rho\left(\vec{x}^{\prime}\right)=-\frac{1}{2} z^{\Delta-d} \delta \phi^{T} \mathbf{A} \rho
$$

In order to have a classical solution we add to the action a boundary term to cancel (2.16) giving:

$$
\begin{aligned}
\mathcal{S} & =\frac{1}{2} \int d^{d+1} x \sqrt{g} \phi\left[-\nabla^{2}+m^{2}\right] \phi+\frac{1}{2} \int_{z=\epsilon} d^{d} x d^{d} x^{\prime} z^{\Delta-d} \phi(\vec{x}, z) \mathbf{A}\left(\vec{x}, \vec{x}^{\prime}\right) \rho\left(\vec{x}^{\prime}\right)= \\
& =\mathcal{S}_{0}+\frac{1}{2} \epsilon^{\Delta-d} \phi^{T} \mathbf{A} \rho
\end{aligned}
$$

Evaluating (2.17) on a classical solution $\phi_{c l}$ one gets:

$$
\mathcal{S}\left[\phi_{c l}\right]=\frac{1}{2} \epsilon^{\Delta-d} \phi_{c l}^{T} \mathbf{A} \rho
$$

It turns out that a convenient choice reproducing the usual form of the two point function in the $\epsilon \rightarrow 0$ limit is:

$$
\begin{aligned}
& \boldsymbol{\Omega}_{\vec{x}^{\prime}}^{\overrightarrow{\vec{x}}} \equiv \Delta_{+} \delta_{\vec{x}^{\prime}}^{\vec{x}} \\
& \mathbf{A}_{\vec{x}^{\prime}}^{\overrightarrow{x^{\prime}}} \equiv-2 \Delta_{+} \frac{\epsilon^{\Delta_{+}-\Delta_{-}+2}}{\left(\epsilon^{2}+\left|\vec{x}-\vec{x}^{\prime}\right|^{2}\right)^{\Delta_{+}+1}} .
\end{aligned}
$$

Note, using (2.6) that $\mathbf{A}$ is proportional to a delta function as $\epsilon \rightarrow 0$. This choice is convenient because it assures that the solution:

$$
\phi_{\rho}(\vec{x}, z)=\int d^{d} x^{\prime} \frac{\rho\left(\vec{x}^{\prime}\right) z^{\Delta_{+}}}{\left(z^{2}+\left|\vec{x}-\vec{x}^{\prime}\right|^{2}\right)^{\Delta_{+}}},
$$

satisfies the boundary condition:

$$
\left.z^{-\Delta_{-}}\left(z \partial_{z}-\Delta_{+}\right) \phi(\vec{x}, z)\right|_{z=\epsilon}=\mathbf{A}_{\vec{x}^{\prime}}^{\vec{x}} \rho^{\vec{x}^{\prime}}
$$


at any finite $\epsilon$ by construction.

Finally, evaluating (2.17) on the classical solution (2.20) we get

$$
\mathcal{S}[\rho]=\frac{1}{2} \int_{z=\epsilon} d^{d} x d^{d} x^{\prime} d^{d} x^{\prime \prime} \frac{\rho\left(\vec{x}^{\prime}\right) \mathbf{A}\left(\vec{x}, \vec{x}^{\prime \prime}\right) \rho\left(\vec{x}^{\prime \prime}\right)}{\left(z^{2}+\left|\vec{x}-\vec{x}^{\prime}\right|^{2}\right)^{\Delta_{+}}} .
$$

This gives the desired result when we remove the cutoff:

$$
\mathcal{S}[\rho]=-\pi^{\frac{d}{2}} \frac{\Gamma\left(\Delta_{+}+1-\frac{d}{2}\right)}{\Gamma\left(\Delta_{+}\right)} \int d^{d} x d^{d} x^{\prime} \frac{\rho(\vec{x}) \rho\left(\vec{x}^{\prime}\right)}{\left|\vec{x}-\vec{x}^{\prime}\right|^{2 \Delta_{+}}} .
$$

Note that using this formalism the degenerate case $\Delta_{+}=\Delta_{-}=\frac{d}{2}$ does not pose any problem since the exponent $\Delta($ see $(2.6))$ is shifted by one in $(2.19)$ and becomes $\Delta+1$. The unitarity bound now emerges naturally at $\Delta=\frac{d-2}{2}$ where a logarithmic divergence appears in $(2.22)$.

\section{3. $A d S_{d+1} / C F T_{d}$ deformed by a double trace operator.}

In this section we will discuss the deformation in the gravity side caused by turning on a double trace deformation $\int d^{d} x \mathcal{O}^{2}$ in the dual CFT, where $\mathcal{O}$ is an operator of dimension $d / 2$. A "Double trace" deformation is a special case of the class of "multi trace" deformations discussed in [1], and the same method described here can also be applied to this more general case. Also, in what follows we assume that the gravity field $\phi$ which couples to the boundary operator is free. This does not restrict our discussion in any important way as interaction vertices can be easily incorporated without changing our basic conclusions.

\subsection{The change in the boundary conditions}

As described in [1], One can deform the $d$-dimensional dual CFT with "double trace" operators by introducing an auxiliary field $\lambda$. Keeping a source term for $\mathcal{O}$ the expression is

$$
e^{\frac{\tilde{h}}{2} \int \mathcal{O}^{2}+\int \rho \mathcal{O}}=\int D[\lambda] e^{\int(\lambda+\rho) \mathcal{O}-\int \frac{1}{2 \tilde{h}} \lambda^{2}}=\int D[\lambda] e^{\int \lambda \mathcal{O}-\int \frac{1}{2 \tilde{h}}(\lambda-\rho)^{2}},
$$

where we shifted variables $\lambda \rightarrow \lambda+\rho$ to go to the last expression. The generating functional in the presence of the double trace operator deformation is therefore

$$
Z_{C F T}^{\text {d.t. }}[\rho] \equiv\left\langle e^{\frac{\tilde{h}}{2} \int \mathcal{O}^{2}+\int \rho \mathcal{O}}\right\rangle=\int D[\lambda] e^{-\frac{1}{2 \tilde{h}} \int(\lambda-\rho)^{2}} Z[\lambda]_{C F T}
$$


where $Z[\lambda] \equiv\left\langle e^{\int \lambda \mathcal{O}}\right\rangle$ and the notation $\langle\ldots\rangle$ means the path integral in the CFT with the undeformed action.

We now use (2.1) in the classical gravity approximation in order to move to the gravity side and get:

$$
Z_{C F T}^{\text {d.t. }}[\rho]=\int D[\lambda] e^{-\frac{1}{2 \tilde{h}} \int_{\partial}(\lambda-\rho)^{2}} Z[\lambda]_{C F T}=\int D[\lambda] D[\phi ; \lambda] e^{-\frac{1}{2 \tilde{h}} \int(\lambda-\rho)^{2}} e^{-\mathcal{S}_{g r}[\phi]}=Z_{g r}^{\text {d.t. }}[\rho] .
$$

We will use below these expressions to define a new gravity action by

$$
Z_{g r}^{\text {d.t. }}[\rho]=\int D[\phi] e^{-S_{g r}[\phi ; \rho ; \tilde{h}]},
$$

where the path integral is carried out on all $\phi$ without any boundary conditions (this will be elaborated below).

We begin by rewriting (3.3) with the boundary condition on $\phi$ which now reads

$$
\epsilon^{-\frac{d}{2}}\left(\left(z \partial_{z}-\frac{d}{2}\right) \phi\right)_{z=\epsilon}=\mathbf{A} \lambda
$$

imposed by a delta function

$$
Z_{g r}^{\text {d.t. }}[\rho]=\int D[\lambda] D[\phi] e^{-\frac{1}{2 h} \int(\lambda-\rho)^{2}} e^{-\mathcal{S}_{g r}[\phi]} \prod_{\vec{x}} \delta\left(\left.z^{-\frac{d}{2}}\left(z \partial_{z}-d / 2\right) \phi(\vec{x}, z)\right|_{z=\epsilon}-(\mathbf{A} \lambda)(\vec{x})\right) .
$$

The product of delta functions for each point on the boundary can be represented by an integral on an additional boundary field $\beta(\vec{x})$ as follows:

$$
Z_{g r}^{\text {d.t. }}[\rho]=\int D[\phi] D[\lambda] D[\beta] e^{-S[\phi, \lambda, \beta ; \rho, \tilde{h}]},
$$

where now $\lambda, \phi, \beta$ are independent and we denoted:

$$
\begin{aligned}
& S[\phi, \lambda, \beta ; \rho, \tilde{h}]= \\
& =\frac{1}{2} \int_{B u l k} d^{d+1} x \sqrt{g} \phi\left(-\nabla^{2}-\frac{d^{2}}{4}\right) \phi+\frac{1}{2} \int_{z=\epsilon} d^{d} x d^{d} x^{\prime} z^{-d} \phi(\vec{x}, z)\left(z \partial_{z}-\frac{d}{2}\right) \phi(\vec{x}, z) \\
& +\frac{1}{2 \tilde{h}} \int_{z=\epsilon} d^{d} x(\lambda-\rho)^{2}(\vec{x})+\frac{i}{2} \int_{z=\epsilon} \beta(\vec{x})\left[\left(z \partial_{z}-d / 2\right) \phi(\vec{x}, z)-z^{\frac{d}{2}}(\mathbf{A} \lambda)(\vec{x})\right]= \\
& =\mathcal{S}_{0}+\frac{1}{2} \epsilon^{-d} \phi^{T}\left(z \partial_{z}-\frac{d}{2}\right) \phi+\frac{1}{2 \tilde{h}}(\lambda-\rho)^{T}(\lambda-\rho)+\frac{i}{2} \beta^{T}\left[\left(z \partial_{z}-\frac{d}{2}\right) \phi-z^{\frac{d}{2}} \mathbf{A} \lambda\right] .
\end{aligned}
$$

We first notice that the $\lambda$ integral is Gaussian, integrating over it we get:

$$
Z_{g r}^{d . t .}[\rho]=\int D[\phi] D[\beta] e^{-\mathcal{S}[\phi, \beta ; \rho, \tilde{h}]},
$$


where

$$
\mathcal{S}[\phi, \beta ; \rho, \tilde{h}]=\mathcal{S}_{0}+\frac{1}{2} \epsilon^{-d} \phi^{T}\left(z \partial_{z}-\frac{d}{2}\right) \phi+\frac{\tilde{h}}{8} \epsilon^{d} \beta^{T} \mathbf{A}^{2} \beta+\frac{i}{2} \beta^{T}\left(\left(z \partial_{z}-\frac{d}{2}\right) \phi-z^{\frac{d}{2}} \mathbf{A} \rho\right) .
$$

Now the $\beta$ integral also became a Gaussian integral so we can perform it and get:

$$
Z_{g r}^{d . t .}[\rho]=\int D[\phi] e^{-\mathcal{S}[\phi ; \rho, \tilde{h}]},
$$

where

$$
\begin{aligned}
& \mathcal{S}[\phi ; \rho, \tilde{h}]=\mathcal{S}_{0}+ \\
& +\frac{1}{2} \epsilon^{-d} \phi^{T}\left(z \partial_{z}-\frac{d}{2}\right) \phi+\frac{1}{2 \tilde{h}} \epsilon^{-d}\left(\left(z \partial_{z}-\frac{d}{2}\right) \phi-z^{\frac{d}{2}} \mathbf{A} \rho\right)^{T} \mathbf{A}^{-2}\left(\left(z \partial_{z}-\frac{d}{2}\right) \phi-z^{\frac{d}{2}} \mathbf{A} \rho\right) .
\end{aligned}
$$

Since we integrated over the boundary condition we now have an action without any boundary conditions. We can now ask what are the classical solutions of this action. The linear variation is:

$$
\begin{aligned}
\delta \mathcal{S} & =-\int d^{d+1} x \sqrt{g} \delta \phi\left(\nabla^{2}+\frac{d^{2}}{4}\right) \phi+ \\
& +\frac{1}{\tilde{h}} \epsilon^{-d}\left(\tilde{h} \mathbf{A}^{2} \phi+\left(z \partial_{z}-\frac{d}{2}\right) \phi-\epsilon^{\frac{d}{2}} \mathbf{A} \rho\right)^{T} \mathbf{A}^{-2}\left(z \partial_{z}-\frac{d}{2}\right) \delta \phi .
\end{aligned}
$$

The classical solutions are now characterized by the usual equations of motion in the bulk of $A d S$ plus a "boundary equation of motion":

$$
\epsilon^{-\frac{d}{2}}\left(\left(z \partial_{z}-\frac{d}{2}\right) \phi+\tilde{h} \mathbf{A}^{2} \phi\right)_{z=\epsilon}=\mathbf{A} \rho
$$

Equations (3.12) and (3.14) are the main results of the paper. We obtained that the path integral in the theory deformed by the double trace deformation is a path integral without boundary conditions on $\phi$ but with a modified boundary action, which effectively imposes new boundary conditions on classical solutions, as can be seen by comparing (3.14) with the boundary condition (3.5).

\subsection{The induced change to the 2-point function}

We would now like to use this formulation to extract the change to 2-point functions of the operator $\mathcal{O}$ on the gravity side. This will match exactly the results expected from the field theory. 
Evaluating (3.12) on the classical solution obeying (3.14) we get again (see (2.18)):

$$
\mathcal{S}\left[\phi_{c l} ; \rho, \tilde{h}\right]=\frac{1}{2} \epsilon^{-\frac{d}{2}} \phi_{c l}^{T} \mathbf{A} \rho .
$$

We will solve the equations of motion in the bulk and on the boundary iteratively. Expanding $\phi$ in powers of $\tilde{h}$ we write:

$$
\phi(\vec{x}, z)=\sum_{n=0}^{\infty} \tilde{h}^{n} \phi^{(n)}(\vec{x}, z)=\phi^{(0)}+\tilde{h} \phi^{(1)} \ldots
$$

Plugging this expression into (3.14) we get:

$$
\left.\epsilon^{-\frac{d}{2}}\left(\left(z \partial_{z}-\frac{d}{2}\right)+\tilde{h} \mathbf{A}^{2}\right)\left(\phi^{(0)}+\tilde{h} \phi^{(1)} \ldots\right)\right|_{z=\epsilon}=\mathbf{A} \rho .
$$

The zeroth order equation is:

$$
\left.\epsilon^{-\frac{d}{2}}\left(z \partial_{z}-\frac{d}{2}\right) \phi^{(0)}\right|_{z=\epsilon}=\mathbf{A} \rho .
$$

The solution to that is exactly (2.20). The higher order equations are:

$$
\begin{aligned}
\left.\left(z \partial_{z}-\frac{d}{2}\right) \phi^{(1)}(\vec{x}, z)\right|_{z=\epsilon} & =-\mathbf{A}^{2} \phi^{(0)}(\vec{x}, \epsilon) \\
& : \\
\left.\left(z \partial_{z}-\frac{d}{2}\right) \phi^{(n+1)}(\vec{x}, z)\right|_{z=\epsilon} & =-\mathbf{A}^{2} \phi^{(n)}(\vec{x}, \epsilon)
\end{aligned}
$$

Since $\mathbf{A} \rho$ does not depend on $z$, the solution to the $(\mathrm{n}+1)^{\prime}$ th order equation is the same as (2.20) only with $\rho \rightarrow-\epsilon^{-\frac{d}{2}} \mathbf{A} \phi^{(n)}(\vec{x}, \epsilon)$. so in particular:

$$
\begin{aligned}
\phi^{(1)}(\vec{x}, z) & =-\int d^{d} x^{\prime} \epsilon^{-\frac{d}{2}} \mathbf{A} \phi^{(0)}\left(\vec{x}^{\prime}, \epsilon\right) \frac{z^{\frac{d}{2}}}{\left(z^{2}+\left|\vec{x}-\vec{x}^{\prime}\right|^{2}\right)^{\frac{d}{2}}}= \\
& =-\int d^{d} x^{\prime} d^{d} x^{\prime \prime} d^{d} x^{\prime \prime \prime} \frac{z^{\frac{d}{2}} A\left(\vec{x}^{\prime}, \vec{x}^{\prime \prime}\right) \rho\left(\vec{x}^{\prime \prime \prime}\right)}{\left(z^{2}+\left|\vec{x}-\vec{x}^{\prime}\right|^{2}\right)^{\frac{d}{2}}\left(\epsilon^{2}+\left|\vec{x}^{\prime \prime}-\vec{x}^{\prime \prime \prime}\right|^{2}\right)^{\frac{d}{2}}} .
\end{aligned}
$$

(recall that $\Delta_{+}=d / 2$ ). As before, let us evaluate (3.15) to first order in $\tilde{h}$ by plugging in (3.20):

$$
\begin{aligned}
\mathcal{S}\left[\phi_{c l} ; \epsilon\right] & =\int d^{d} x_{1} d^{d} x_{2} d^{d} x_{3} \frac{\rho\left(\vec{x}_{1}\right) A\left(\vec{x}_{2}, \vec{x}_{3}\right) \rho\left(\vec{x}_{3}\right)}{\left(\epsilon^{2}+\left|\vec{x}_{1}-\vec{x}_{2}\right|^{2}\right)^{\frac{d}{2}}}- \\
& -\tilde{h} \int d^{d} x_{1} \ldots d^{d} x_{5} \frac{\rho\left(\vec{x}_{1}\right) A\left(\vec{x}_{2}, \vec{x}_{3}\right) A\left(\vec{x}_{4}, \vec{x}_{5}\right) \rho\left(\vec{x}_{5}\right)}{\left(\epsilon^{2}+\left|\vec{x}_{1}-\vec{x}_{2}\right|^{2}\right)^{\frac{d}{2}}\left(\epsilon^{2}+\left|\vec{x}_{3}-\vec{x}_{4}\right|^{2}\right)^{\frac{d}{2}}}+\mathcal{O}\left(\tilde{h}^{2}\right) .
\end{aligned}
$$


When we now take $\epsilon \rightarrow 0$ and use (2.6) we get:

$$
\begin{gathered}
\mathcal{S}\left[\phi_{c l}\right]=\frac{-d \pi^{\frac{d}{2}}}{\frac{d}{2} !} \int d^{d} x_{1} d^{d} x_{2} \frac{\rho\left(\vec{x}_{1}\right) \rho\left(\vec{x}_{2}\right)}{\left|\vec{x}_{1}-\vec{x}_{2}\right|^{d}}- \\
-\tilde{h}\left(\frac{-d \pi^{\frac{d}{2}}}{\frac{d}{2} !}\right)^{2} \int d^{d} x_{1} d^{d} x_{2} d^{d} x_{3} \frac{\rho\left(\vec{x}_{1}\right) \rho\left(\vec{x}_{3}\right)}{\left|\vec{x}_{1}-\vec{x}_{2}\right|^{d}\left|\vec{x}_{2}-\vec{x}_{3}\right|^{d}}+\mathcal{O}\left(\tilde{h}^{2}\right) .
\end{gathered}
$$

This is the term that upon varying twice with respect to $\rho$ gives the right leading $\tilde{h}$ correction to the two point function in the CFT in the limit $\epsilon \rightarrow 0$.

$$
\begin{aligned}
<\mathcal{O}(\vec{x}) \mathcal{O}(\vec{y})>=\frac{1}{|\vec{x}-\vec{y}|^{d}} & +\tilde{h} \int d^{d} u \frac{1}{|\vec{x}-\vec{u}|^{d}|\vec{u}-\vec{y}|^{d}}+ \\
& +\tilde{h}^{2} \int d^{d} u d^{d} v \frac{1}{|\vec{x}-\vec{u}|^{d}|\vec{u}-\vec{v}|^{d}|\vec{v}-\vec{y}|^{d}}+\ldots,
\end{aligned}
$$

as in e.g. equation (2.4) in [1]. It is also clear that the higher order corrections will follow the same pattern, where in each order one adds another boundary integration and another boundary-to-boundary propagator.

It is worth revisiting one point. (3.14) is an equation of motion on the boundary and not a boundary condition like (2.15). The important difference between these two is that boundary conditions restrict the quantum fluctuations one is integrating over while an equation of motion on the boundary does not. One might ask whether we should also impose (3.14) as a boundary condition in the quantum path integral. At the level that we analyzed the 2-point functions we can not answer this question since we used only the classical configurations. Only when we start considering quantum loops in $A d S$ will the difference between the two formulations appear.

\subsection{Renormalization - the CFT side.}

The expansion of the two point function in the CFT deformed by the double trace operator is of the form (3.23). Notice that already the first correction diverges when the intermediate coordinate $\vec{u}$ approaches either $\vec{x}$ or $\vec{y}$, and the following orders diverge correspondingly, so one needs to regularize these expressions. This can be done by renormalizing the operator $\mathcal{O}$ as follows: First one introduces an ultraviolet cutoff $\epsilon$ which prevents $\vec{u}$ from approaching either $\vec{x}$ or $\vec{y}$ too closely, which can be done by changing the propagator to:

$$
<\mathcal{O}(\vec{x}) \mathcal{O}(\vec{y})>=\frac{1}{\left(\epsilon^{2}+|\vec{x}-\vec{y}|^{2}\right)^{\frac{d}{2}}}+\tilde{h} \int \frac{d^{d} u}{\left(\epsilon^{2}+|\vec{x}-\vec{u}|^{2}\right)^{\frac{d}{2}}\left(\epsilon^{2}+|\vec{u}-\vec{y}|^{2}\right)^{\frac{d}{2}}}+\ldots
$$


where the specific form we chose for the cutoff is suggested by the $A d S$ dual but in the end of the day we will anyways take it to zero (and be left with cutoff independent answers).

It is easy to identify the divergent part of the integral:

$$
\begin{aligned}
\tilde{h} \int \frac{d^{d} u}{\left(\epsilon^{2}+|\vec{x}-\vec{u}|^{2}\right)^{\frac{d}{2}}\left(\epsilon^{2}+|\vec{u}-\vec{y}|^{2}\right)^{\frac{d}{2}}}= \\
\quad=\frac{\tilde{h}}{|\vec{x}-\vec{y}|^{d}}\left(2 \Omega_{d} \log \left(\frac{|\vec{x}-\vec{y}|}{\epsilon}\right)+\text { finite }+O\left(\frac{\epsilon}{|\vec{x}-\vec{y}|}\right)\right),
\end{aligned}
$$

where $\Omega_{d}$ is the volume of the $d-1$ unit sphere.

This phenomenon is familiar in field theory and is usually dealt with by renormalizing the operator $\mathcal{O}$ order by order in conformal perturbation theory [11]. We thus define a renormalized operator $\widehat{\mathcal{O}}$ as follows:

$$
\widehat{\mathcal{O}}(\vec{x}) \equiv \mathcal{O}(\vec{x})\left(1-\tilde{h} \Omega_{d} \log \left(\frac{\Lambda}{\epsilon}\right)\right)
$$

where $\Lambda$ is an arbitrary scale needed because we are renormalizing $\mathcal{O}(\vec{x})$ which can not depend on $\vec{y}$.

Clearly in the two point function of $\widehat{\mathcal{O}}$ no divergences appear to first order in $\tilde{h}$ and one gets:

$$
\begin{aligned}
<\widehat{\mathcal{O}}(\vec{x}) \widehat{\mathcal{O}}(\vec{y})> & =\frac{1}{|\vec{x}-\vec{y}|^{d}}-\tilde{h} \frac{2 \Omega_{d} \log \left(\frac{\Lambda}{\epsilon}\right)}{|\vec{x}-\vec{y}|^{d}}+\tilde{h} \int \frac{d^{d} u}{\left(\epsilon^{2}+|\vec{x}-\vec{u}|^{2}\right)^{\frac{d}{2}}\left(\epsilon^{2}+|\vec{u}-\vec{y}|^{2}\right)^{\frac{d}{2}}}+O\left(\tilde{h}^{2}\right) \\
& =\frac{1}{|\vec{x}-\vec{y}|^{d}}+\tilde{h} \frac{2 \Omega_{d} \log \left(\frac{|\vec{x}-\vec{y}|}{\Lambda}\right)}{|\vec{x}-\vec{y}|^{d}}+\tilde{h} \frac{\text { finite }}{|\vec{x}-\vec{y}|^{d}}+O\left(\tilde{h}^{2}\right) .
\end{aligned}
$$

Note that to first order in $\tilde{h}$ this looks like a correction to the dimension of $\mathcal{O}$. However the perturbation actually breaks conformal invariance as can be seen by the following simple argument:10 The same calculation can now be performed to compute corrections to the two point function of the double trace deformation itself, i.e. $\left\langle\mathcal{O}^{2}(\vec{x}) \mathcal{O}^{2}(\vec{y})\right\rangle$, showing that the same kind of logarithmic divergences appear, breaking conformal invariance. Alternatively one can use the Callan-Symanzik equation to see that the beta function is no longer zero.

10 We thank D.Kutasov for pointing this out for us. 


\subsection{Renormalization - the gravity side.}

The divergences we encountered on the CFT side appear also in the gravity side. Indeed if we want to have a sensible description of the deformations caused by the insertion of the double trace operator, we must see that the deformed field configuration (3.16) is finite. Since the corrections to $\phi$ produce via (3.15) the corrections to the gravity action (which in turn change the two point function) one can easily see that the correction to first order in $\tilde{h}$ (3.20) diverges in exactly the same way as (3.25). The reason for this divergence is that the "source" of $\phi^{(1)}$ which is $\epsilon^{-\frac{d}{2}} \mathbf{A} \phi^{(0)}(\vec{x}, \epsilon)$ diverges. We now show how to regularize the gravity action so as to get a finite answer for (3.20) and then show that this regularization could have been easily "guessed" from the CFT regularization in the previous section. The way we chose to regularize the gravity theory is by changing the boundary condition (3.14) to:

$$
\epsilon^{-\frac{d}{2}}\left(\left(z \partial_{z}-\frac{d}{2}\right) \phi+\tilde{h} \mathbf{A}^{2} \phi\right)=\mathbf{A}\left(1-\tilde{h} \Omega_{d} \log \left(\frac{\Lambda}{\epsilon}\right)+O\left(\tilde{h}^{2}\right)\right) \rho .
$$

The zeroth order equation remains the same as (3.18) and we rewrite (2.20) in matrix notations for brevity as $\phi^{(0)}(\vec{x}, z)=\mathbf{B}^{z} \rho$. The first order equation changes upon regularization to:

$$
\begin{aligned}
& \left.\epsilon^{-\frac{d}{2}}\left(z \partial_{z}-\frac{d}{2}\right) \phi^{(1)}(\vec{x}, z)\right|_{z=\epsilon}=-\mathbf{A}\left(\mathbf{A} \epsilon^{-\frac{d}{2}} \phi^{(0)}(\vec{x}, \epsilon)+\Omega_{d} \log \left(\frac{\Lambda}{\epsilon}\right) \rho(\vec{x})\right)= \\
= & -\mathbf{A}\left(\mathbf{A} \epsilon^{-\frac{d}{2}} \mathbf{B}^{\epsilon}+\Omega_{d} \log \left(\frac{\Lambda}{\epsilon}\right)\right) \rho .
\end{aligned}
$$

This way we simply removed from the source of $\phi^{(1)}$ the divergent part, making it finite, and when computing now the two point function we will get the correct finite expression (3.27) to first order in $\tilde{h}$. One could have "guessed" this correction by the following argument: The source term for $\mathcal{O}$ in the CFT is $\int \rho \mathcal{O}$. Since the "physical" operator is the renormalized one (3.26) the source term should be changed to:

$$
\int d^{d} x \rho(\vec{x}) \widehat{\mathcal{O}}(\vec{x})=\int d^{d} x \rho(\vec{x})\left(1-\tilde{h} \Omega_{d} \log \left(\frac{\Lambda}{\epsilon}\right)+O\left(\tilde{h}^{2}\right)\right) \mathcal{O}(\vec{x}),
$$

thus following the same calculations done in section 2 we will get the new form of the boundary condition (3.28). It is easy to get convinced that this procedure can be continued to all orders in $\tilde{h}$ in both sides of the correspondence simultaneously. 


\section{Spacetime singularities and non-local worldsheets}

11 We have seen in the previous section that the effect of the double trace deformation is to change the boundary conditions on the fields in $A d S$. Given that determining boundary conditions (and boundary terms) in General Relativity, especially at singularities, is a subtle yet crucial step in any actual computation, this is a significant change.

The modification to the worldsheet formulation [1] is even more dramatic - the worldsheet field theory becomes explicitly non-local, with an action of the form

$$
S=\int d^{2} z \mathcal{L}_{0}+\tilde{h} \Sigma_{I, J} c_{I J} \iint d^{2} z_{1} d^{2} z_{2} V^{(I)}\left(z_{1}, \bar{z}_{1}\right) V^{(J)}\left(z_{2}, \bar{z}_{2}\right),
$$

where $\mathcal{L}_{0}$ is a local functional of the worldsheet fields, the $V$ 's are vertex operators on the worldsheet, and $c_{I J}$ are specific numbers. The 2nd term on the RHS of (4.1) is an explicitly non-local contribution to the action, which directly couples the fields at two separate points $\left(z_{1}, \bar{z}_{1}\right)$ and $\left(z_{2}, \bar{z}_{2}\right)$ on the worldsheet.

In this section we will attempt to speculate on the relation of these two facts. We will suggest that

1. The phenomena of non-local worldsheet theories might occur in a much wider class of stringy backgrounds,

2. In many cases the non-localities would be of an even more severe type than (4.1),

3. This behavior will be associated with singularities or boundaries in spacetime (we of course understand many kinds of singularities in String theory, but hardly all).

Unfortunately since we do not yet have a concrete workable example 12 , this section is highly speculative.

This might put in a broader context the results of the previous section that the deformation effects the boundary conditions. If the non-local deformation of the worldsheet is associated with submanifolds of spacetime (boundaries or singularities) it can only manifest there at low energies as boundary conditions 13 .

More precisely, we will suggest that the boundaries/singularities associated with a non-local worldsheet are ones at finite distance in spacetime. AdS certainly falls into that

11 Parts of this section were developed in collaboration with E. Silverstein

12 In a "work in progress" project we are trying to develop such examples

13 This, however, immediately implies effects in the bulk, away from the boundary/singularity, as processes can communicate with the boundary/singularity. This should perhaps be viewed as "finite volume/distance effects". Reference [2] clearly exhibits such effects. 
category as particles with appropriate masses can propagate to the boundary of spacetime and come back at finite time - they view the boundary as a finite distance defect from which they scatter.

To justify these conjectures we begin by discussing whether there are any reasons why the worldsheet has to be local. After all, it is an auxiliary object with, for the most part 14 , only indirect consequences for space time. One answer is that it works remarkable well, and might therefore be treated as an axiom. A more substantive answer is that taking the worldsheet to be a local theory is "technically natural" for most of the backgrounds studied so far in string theory (which are of the form flat space $\times$ compact manifold). This naturality argument, however, may not prohibit non-local worldsheets for other more complicated backgrounds (such as $A d S$ where we see a departure from worldsheet locality).

By worldsheet locality being "technically natural" we refer to the Fischler-Susskind mechanism [14,15]. For completeness we will briefly review this mechanism: consider string theory on some tachyon free (at tree level) non-supersymmetric background with a 1-loop induced dilaton tadpole. Other computations on the torus, and certainly at higher genus, would be divergent because of this tadpole. An example of this, given in [14], is of an npoint function of vertex operators on a torus. The divergence comes from the boundary of the moduli space of the punctured torus where it looks like a sphere with an infinitesimal handle, well separated from the vertex operators. The diagram then factorizes into a tadpole of the dilaton at zero momentum on an infinitesimal torus, an $n+1$-point function on the sphere involving the $\mathrm{n}$ operators we had before + the dilaton vertex operator, and a dilaton propagator between these 2 components. It is then shown in [14, 15] that this region leads to a divergence in the n-point function.

Fortunately one can "renormalize" this divergence, at the price of shifting the background to that of a cosmological constant - this is the Fischler-Susskind mechanism. This is done by adding a divergent counterterm to the worldsheet theory already at the sphere level. The main point is that because this counterterm originates from a torus which looks like an infinitesimal handle attached to a sphere, it yields a local correction to the worldsheet action. Hence the worldsheet locality is preserved under quantum renormalization of the theory - it is "technically natural".

We can now discuss when a violation of worldsheet locality might be forced upon

14 with notable exceptions, such as the interpretation of macroscopic strings in AdS/CFT as Wilson lines [12,13]. 
us. An important point in the discussion above was that the divergence was associated with an on-shell dilaton tadpole. This was used for example when factorizing the diagram to separate the small handle from the vertex operators in the diagrams, which was an essential step in renormalizing the theory. A particle going on-shell implies an that it can propagate for a long time in spacetime. Hence we expect not to be able to factor out the small handles when the divergences are associated with a singularity or a defect in spacetime which particles can reach at finite time. In this case, it might be that one will encounter a divergence on the torus, but one will not be able to isolate the singularity, as we did before, as associated with a distinct very small handle. Rather the divergence will be associated with a torus at a finite modular parameter. If the theory is to make sense, one needs to absorb this divergences on the sphere. Even if this is possible, it will be done not by an infinitesimal handle attached to a sphere, but rather by a macroscopic handle. This will make the worldsheet theory on the sphere non-local.

It seems that one can therefore suggest that divergences associated with finite distance/time processes in spacetime will be reflected in string theory by having a non-local worldsheet. We do not however have a concrete example of this type 15 , hence it is difficult to really check this conjecture. We will therefore turn our attention to another speculative example, that of string theory on Rindler space 16 , which although far from being understood, points to the same direction. Let us adopt the coordinate system in which the Rindler metric is static (we follow the conventions of [17)

$$
d s^{2}=e^{2 z}\left(-d t^{2}+d z^{2}\right)
$$

The Rindler horizon is at $z \rightarrow-\infty$. As a first guess one might try and write down the string worldsheet action using the metric (4.2). However, since the worldsheet theory is target-space reparametrization invariant, the action is the same as part of flat space, and there is no suppression in the worldsheet path integral for a string trying to cross the Rindler Horizon. However, we do not want to allow the string to do so because

1. the accelerating Rindler observer can not receive information from behind the horizon.

15 It seems that one might be able to build models that have this property. They are associated with non-trivial singular cosmologies. Other relations of non-local worldsheets to cosmology are being studied in [16].

16 Parts of this analysis were developed jointly with E. Silverstein. This example is intimately related to that of particle creation in cosmological setting which will be elaborated in [16]. 
2. If we re-enstate the entire spacetime we will be forced to the ordinary Minkowski vertex operators and Minkowski vacuum, and will end up with ordinary Minkowski space.

Hence it seems that we have reached an impasse.

To try and make progress we note that the problem has a similar flavor to the one we have been discussing - there is a boundary (more generally - some defect) in spacetime which the particles can reach at finite proper distance, and where one needs to define the boundary conditions (this is one of the differences between a quarter of Minkowski space and Rindler space). Let us therefore try to apply the ideas mentioned above about non-local worldsheets. Again unfortunately, the picture that we will present will be very incomplete but, we believe, suggestive and in line with the discussion above of worldsheet non-localities.

We want to reach a point where we discuss only the part of the worldsheet outside the horizon, and measurement processes outside the horizon. As explained above, however, the worldsheet can easily cross the horizon without any suppression in the action. We will therefore organize the perturbation theory in the following way. We will split the space of the configurations of the worldsheet - the measure in the worldsheet path integral - into different regions depending on how many times the worldsheet crosses the horizon. Hence a sphere which has $k$ prongs going into the horizon will be part of the region associated with a sphere with $k$ holes (see figure 1). Similarly a torus which goes from one side of the horizon to the other looks to the outside observer as a sphere with 2 holes (figure 2). After splitting the diagram over what the outside observer sees, one performs the path integral over the region behind the horizon (we will not require the details at the level of the discussion here).

When we have a boundary where the string worldsheet crosses the horizon (shaped like a circle on the worldsheet) we need to specify the boundary conditions there. The standard way of doing so is by specifying the closed string state along each of the circles. Let us first consider the diagram in figure 1. Since each of the prongs that goes behind the horizon is independent from the others, the states on the different boundary circles are not correlated. The path integral is therefore carried out with some "boundaries multi-state"

$$
\Pi_{i}\left(\Sigma_{\left(I_{i}\right)} C_{i}^{\left(I_{i}\right)} \mid \phi^{\left(I_{i}\right)}>_{\gamma_{i}}\right)
$$

where $\mid \phi^{(I)}>$ run on all the states in the closed string channel as the index $I$ varies, 


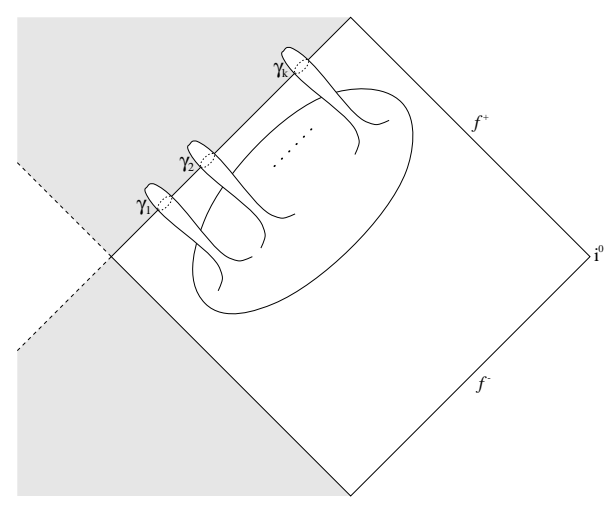

Figure 1: A sphere diagram in Rindler space with k prongs reaching beyond the horizon

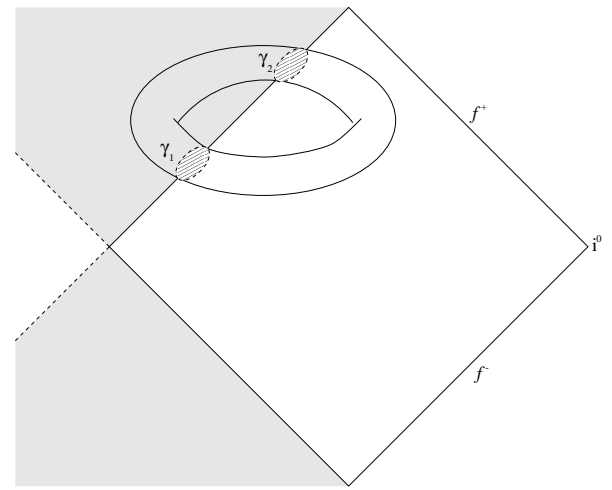

Figure 2: A torus diagram in Rindler space which crosses the horizon along 2 circles

and $C_{i}^{(I)}$ are some numbers 17 . This is still to be considered a local deformation of the worldsheet since the states in different points of the worldsheets - the different circles - are uncorrelated.

The situation is different in figure 2 (torus crossing the horizon) - since the 2 circles are connected behind the horizon, the 2 states on the 2 boundary circles will be correlated. One therefore inserts the following "boundaries multi-state":

$$
\Sigma_{(I, J)} C^{(I, J)}\left|\phi^{(I)}>_{\gamma_{1}} \times\right| \phi^{(J)}>_{\gamma_{2}}
$$

The theory has now become non-local on the worldsheet since the behavior at different points is correlated explicitly by hand. Hence we see another example where a defect, in

17 In the case where there is only a simple cap behind the horizon, only states in the conformal block of the identity contribute. 
this case an horizon, at finite distance in spacetime, is potentially giving rise to a non-local worldsheet action.

One can easily see that the arguments leading to equation (4.4) can be applied to any singularity which all the particles can reach without being forced on shell. This is encoded by the fact that all the single closed string states can appear as factors in the sum, or correspondingly that one has an entire circle (which can carry the information about the various states). This is actually a more pathological behavior than we have seen in the $A d S$ case. One can pass, however, from this to the $A d S$ as a special case. An important feature of the boundary of $A d S$ is that only a finite number of particles can approach it in finite time. Hence the sum over the states (4.4) collapses into a sum over a finite number of states. In addition, the worldsheets in AdS are pinched to a point as they reach the boundary [18]. The combination of these two points collapses the more general deformation (4.4) into a sum over a finite number of local vertex operators at different points on the worldsheet, i.e, of the form (4.1).

\section{Acknowledgments}

We would like to thank T. Banks, D. Berman, S. Elitzur, A. Giveon, D. Kutasov, J. Maldacena, E. Martinec, E. Rabinovici, E. Silverstein and L. Susskind for helpful discussions. We are particularly indebted to O. Aharony for collaboration at early stages of this work. M.B. is supported by the Israel-U.S. Binational Science Foundation, the IRF Centers of Excellence program, the European RTN network HPRN-CT-2000-00122, and by the Minerva foundation. A.Sh is supported by a Clore fellowship. 


\section{References}

[1] O. Aharony, M. Berkooz and E. Silverstein, 'Multiple-trace operators and non-local string theories," JHEP 0108 (2001) 006 ,hep-th/0105309.

[2] O. Aharony, M. Berkooz and E. Silverstein, "Non-local String Theories in $A d S_{3} \times S^{3}$ and Stable Non-Supersymmetric Backgrounds", hep-th/0112178

[3] J. Maldacena, "The large $N$ limit of superconformal field theories and supergravity," Adv. Theor. Math. Phys. 2 (1998) 231 [Int. J. Theor. Phys. 38 (1998) 1113] hepth/9711200.

[4] E. Witten, "Anti-de Sitter space and holography," Adv. Theor. Math. Phys. 2 (1998) 253 ,hep-th/9802150.

[5] S. S. Gubser, I. R. Klebanov and A. M. Polyakov, "Gauge theory correlators from non-critical string theory," Phys. Lett. B 428 (1998) 105 ,hep-th/9802109.

[6] O. Aharony, S. S. Gubser, J. Maldacena, H. Ooguri and Y. Oz, "Large $N$ field theories, string theory and gravity," Phys. Rept. 323, 183 (2000) hep-th/9905111.

[7] A. Adams and E. Silverstein, "Closed String TAchyons, AdS/CFT and Large N QCD", Phys. Rev. D64:086001, 2001, ,hep-th/0103220

[8] P. Minces and V. O. Rivelles, "Energy and the AdS/CFT correspondence," JHEP 0112 (2001) 010, hep-th/0110189.

[9] E. Witten, "Multi-trace operators, boundary conditions, and AdS/CFT correspondence," hep-th/0112258.

[10] I. R. Klebanov and E. Witten, "AdS/CFT correspondence and symmetry breaking," Nucl. Phys. B 556 (1999) 89 ,hep-th/9905104.

[11] R. Dijkgraaf, E. Verlinde and H. Verlinde, "On Moduli Spaces Of Conformal Field Theories With $\mathrm{c} \geq 1$," in *Copenhagen 1987, proceedings, perspectives in string theory*, $11 \%-13 \%$.

[12] J. Maldacena, "Wilson Loops in Large N Field Theory", Phys. Rev. Lett. 80, 4859 (1998), hep-th/9803002.

[13] S. J. Rey and J. Yee, "Macroscopic Strings as Heavy Quarks in Large N Gauge Theory and Anti-de-Sitter Supergravity", Eur. Phys. J. C 22, 379 (2001), ,hep-th/9803001.

[14] W. Fischler and L. Susskind, "Dilaton Tadpoles, String Condensates, and Scale Invariance 2." Phys. Lett. B 173, 262, (1986)

[15] W. Fischler and L. Susskind, "Dilaton Tadpoles, String Condensates, and Scale Invariance." Phys. Lett. B 171, 383, (1986)

[16] O. Aharony, M. Berkooz and E. Silverstein, work in progress

[17] N.D. Birrell and P.C.W. Davies, "Quantum Fields in Curved Space", Cambridge Monographs on Mathematical Physics, 1982

[18] J. de Boer, H. Ooguri, H. Robins and J. Tannenhauser, "String theory on $A d S_{3}$," JHEP 9812, 026 (1998), hep-th/9812046. 